Muséologies

Les cahiers d'études supérieures

muséologies

\title{
Acquérir This Situation de Tino Sehgal. Entretiens avec Josée Bélisle, Marie Fraser, Lesley Johnstone et Anne-Marie Zeppetelli
}

\section{Amélie Giguère}

Volume 7, numéro 1, 2014

Le dialogue dans les musées d'art contemporain

URI : https://id.erudit.org/iderudit/1026656ar

DOI : https://doi.org/10.7202/1026656ar

Aller au sommaire du numéro

Éditeur(s)

Association Québécoise de Promotion des Recherches Étudiantes en

Muséologie (AQPREM)

ISSN

1718-5181 (imprimé)

1929-7815 (numérique)

Découvrir la revue

Citer ce document

Giguère, A. (2014). Acquérir This Situation de Tino Sehgal. Entretiens avec Josée Bélisle, Marie Fraser, Lesley Johnstone et Anne-Marie Zeppetelli. Muséologies,

7(1), 225-237. https://doi.org/10.7202/1026656ar

Tous droits réservés (C Association Québécoise de Promotion des Recherches Étudiantes en Muséologie (AQPREM), 2014
Ce document est protégé par la loi sur le droit d'auteur. L'utilisation des services d'Érudit (y compris la reproduction) est assujettie à sa politique d'utilisation que vous pouvez consulter en ligne.

https://apropos.erudit.org/fr/usagers/politique-dutilisation/ 
Entrevue deux

\section{Acquérir This Situation de Tino Sehgal. Entretiens avec Josée Bélisle, Marie Fraser, Lesley Johnstone et Anne-Marie Zeppetelli. \\ Propos recueillis par Amélie Giguère.}


Du 19 mars au 28 avril 2013, le Musée d'art contemporain de Montréal a présenté le travail de Tino Sehgal, un artiste qui recourt à des "interprètes " ou à des "joueurs " pour créer des actions dans les musées et dans les galeries d'art. Pendant les heures d'ouverture du musée, sans interruption, nous avons pu voir dans le hall du rez-de-chaussée la pièce Kiss (2002), minutieusement chorégraphiée pour deux danseurs, dont la trame rappelle quelques baisers célèbres de l'histoire de l'art. Puis, dans une salle à l'étage nous attentait This situation (2007), une action réunissant six intellectuels anglophones et francophones engagés dans des échanges philosophiques.

L'exposition de ces deux " situations construites ", mais surtout l'acquisition par le musée de This situation, ont été le prétexte d'une discussion avec celles qui ont travaillé à la réalisation de ces deux projets. Josée Bélisle, conservatrice responsable de la collection, Marie Fraser, conservatrice en chef et directrice de l'éducation, Lesley Johnstone, conservatrice et commissaire de l'exposition de Tino Sehgal, et Anne-Marie Zeppetelli, archiviste des collections ont accepté de discuter des enjeux de l'acquisition, de la conservation, de la documentation et de la réexposition de cette ouvre atypique fondée sur le langage, l'échange, l'interaction avec le public et le mouvement.

Un premier entretien a eu lieu avec ces quatre personnes le 12 novembre 2012. Un deuxième, avec Lesley Johnstone, s'est tenu le 8 mars 2013, à quelques jours de l'ouverture de l'exposition. Enfin, une dernière rencontre avec Anne-Marie Zeppetelli a eu lieu le 9 mai 2013, soit quelques jours après la clôture de l'exposition. 
Amélie Giguère - Le Musée d'art contemporain de Montréal a fait l'acquisition de This situation de Tino Sehgal. Pouvez-vous me raconter comment est né ce projet d'acquisition?

Marie Fraser - À l'origine de l'acquisition de This situation, il y a un intérêt particulier pour les pratiques qui posent des défis aux musées. Les œuvres de Tino Sehgal sont un excellent exemple de ces pratiques qui nous invitent à revoir nos façons de travailler. Ces dernières années, nous avons toutes suivi son travail, à Berlin, à la Biennale de Venise, au Guggenheim à New York. Puis, nous avons eu envie d'explorer la possibilité d'acquérir une œuvre et de la présenter au musée dans un laps de temps relativement court. Pour nous, il était important que l'acquisition et l'exposition aient lieu à peu près simultanément. Nous connaissions la complexité du travail de Tino Sehgal qui est fondé sur l'oralité, la transmission orale. Pour développer la connaissance de l'œuvre, pour être en mesure de la conserver, de la documenter et de l'exposer, il fallait que nous puissions travailler de concert avec l'artiste dès le moment de l'acquisition.

\section{Avez-vous considéré plusieurs ouvres de Tino Sehgal avant de} faire un choix?

M.F. Oui. Nous avons d'abord abordé la galerie Marian Goodman, qui représente l'artiste, dans le but d'acquérir non pas une œuvre en particulier, mais bien celle qui serait la plus pertinente pour notre collection. J'ai rencontré Karina Daskalov, à New York, qui m'a décrit oralement l'ensemble des œuvres de Tino Sehgal. Pour différentes raisons, notre choix s'est arrêté sur This situation. Je crois que Tino était très excité par cette acquisition. Pour lui, c'est encore une nouvelle expérience, une nouvelle façon de réfléchir à la pérennité de ses œuvres.

Josée Belisle - Après cette rencontre, nous avons cru important de convoquer rapidement le comité de la collection. Dans le cas d'acquisition d'œuvres majeures, nous organisons plusieurs présentations afin de vérifier si le comité est prêt à soutenir notre démarche. Au départ, c'était donc exploratoire; il s'agissait de partager notre désir d'intégrer une œuvre de Tino Sehgal à la collection. Marie a fait une présentation, puis les questions ont fusé de toutes parts! Mais à l'issue de cette première rencontre, nous avions l'aval du comité.

\section{Vous dites que This situation constituait un choix pertinent pour la collection du Musée d'art contemporain de Montréal. Pour quelles raisons?}

M.F. This situation a des dimensions socioéconomique et intellectuelle très fortes. Montréal devenait un lieu d'accueil intéressant, car il y a la présence de deux langues - le français et l'anglais - et une vie culturelle et intellectuelle importante marquée par l'activité de quatre universités. Jusqu'à maintenant, This situation a été présentée surtout en anglais, un peu en allemand, puis en français, à Paris. Nous avons convenu de faire l'acquisition d'une version bilingue de This situation, dont le pourcentage du français et de l'anglais peut être réinterprété en fonction du contexte de présentation de l'œuvre. 
Tino Sehgal a une manière singulière de céder ses œuvres. Pouvez-vous nous raconter comment s'est concrétisée l'acquisition de This situation?

J.B. Karina Daskalov m'a expliqué que nous allions nous réunir autour d'une table et que les conditions attachées à l'acquisition de l'œuvre allaient être énoncées et comprises par tout le monde. Tout allait se faire oralement, aucun document n'allait être échangé. C'est ce qui s'est passé : il y avait la représentante de la galerie, la directrice générale du musée, le président du comité de la collection, la secrétaire générale, un avocat indépendant et nous toutes, Marie, Anne-Marie, Lesley et moi. Karina a exposé le but de la rencontre, puis les cinq conditions. La première condition dit que la présentation de l'œuvre, la première fois, doit être préparée et autorisée par l'artiste ou par son représentant. La deuxième condition rappelle que cette présentation doit avoir lieu pendant les heures d'ouverture du musée et pendant une période qui ne doit pas être inférieure à six semaines.

M.F. La troisième, que les interprètes doivent être rémunérés.

J.B. La quatrième concerne la diffusion de documentation. Comme vous savez, le musée ne peut ni produire ni diffuser des images photographiques ou des images en mouvement de l'œuvre. Il doit aussi s'assurer, dans la mesure du possible, qu'aucune image ne circule. Et la cinquième condition stipule que si le musée - pour des raisons que nous ne pouvons pas imaginer - songe à se départir de cette œuvre, il doit le faire par une transaction orale autorisée par l'artiste ou par son représentant. À l'issue de cette discussion, Karina Daskalov et Paulette Gagnon, la directrice, se sont serré la main et la transaction a été scellée. Tout cela était très spécial. Il y avait une effervescence!

\section{La séance s'est tenue comme il se doit, c'est-à-dire sans papier ni crayon?}

J.B. Sans enregistreuse, sans appareil numérique ni électronique. Inutile de dire que nous savions qu'à la sortie nous allions toutes courir rédiger un mémo électronique pour ne pas oublier ces conditions.

\section{Pourquoi Tino Sehgal explore-t-il cette forme de transaction essentiellement orale?}

J.B. L'oralité est au cœur de sa pratique. Il pousse cette idée jusqu'au bout.

M.F. C'est une oralité consciente de la production des objets et de la circulation des images. Il y a aussi une dimension humaine à tout cela . Tino Sehgal est un artiste qui travaille sur les relations humaines. Il ne veut pas non plus participer à la production matérielle.

Lesley Johnstone J'ajouterais qu'il y a une partie très importante de sa réflexion qui porte sur le musée et ses structures et sur l'économie de l'art. Il réfléchit à la création et à ce qu'il y a autour, la documentation, la médiation, les photos, les communiqués de presse. Les procédures muséales, il les questionne. Le processus d'acquisition fait aussi partie 
de l'œuvre. Le transfert des informations doit se faire de personne à personne. Je pense que tout cela vient de la danse, de sa transmission, de la chorégraphie qui va de corps en corps.

Revenons sur les discussions que vous avez eues dans le cadre de la transaction. Avez-vous pu faire part de vos interrogations, de vos craintes et de vos responsabilités, surtout?

L.J. Nous avons beaucoup discuté du caractère " bilingue " de l'œuvre. Qu'est-ce qu'une version « bilingue » de This situation? Sommes-nous tenues d'assurer que $50 \%$ de la discussion se fasse en français et $50 \%$ en anglais? Si nous prêtons l'œuvre à un musée de Vancouver, par exemple, est-ce que ça peut être $80 \%$ en anglais et $20 \%$ en français? Nous voulions être certaines de pouvoir éventuellement prêter l'œuvre dans des conditions qui soient acceptables pour un musée à l'extérieur de Montréal.

Anne-Marie Zeppetelli Nous avions aussi des interrogations sur la documentation. Le seul document que nous avons reçu, au terme de cette transaction orale, est une facture envoyée par courriel. Pour moi, qui dois enregistrer une transaction, il était primordial de comprendre ce que représente cette exigence de l'artiste. J'ai compris que tous les documents en format numérique peuvent être créés, enregistrés, conservés et attachés à la base de données des collections. Ainsi, chacune de notre côté, après la rencontre, nous avons écrit les cinq conditions, puis nous nous sommes revues, Josée et moi, pour rédiger une sorte de procès-verbal. Ce texte existe dans la base de données. Nous avons donc un dossier électronique et un dossier papier qui ne contient rien. Ça peut paraître étrange, mais ce dossier, qui est numéroté et associé à This situation, témoigne de son acquisition et de sa présence dans la collection.

Vous avez mentionné qu'un avocat a participé à la rencontre. Quel était son rôle?

M.F. Je me souviens que sa première question visait à confirmer que Karina avait bien l'autorité de procéder à la transaction.

J.B. Pour l'avocat, il était aussi très important de comprendre à quel moment le musée deviendrait autonome, c'est-à-dire à quel moment il pourrait présenter l'œuvre sans la participation de Tino Sehgal ou de ses proches collaborateurs.

M.F. La question de l'autonomie revient depuis le début. Le musée a fait cette acquisition dans le but de développer la connaissance de l'œuvre. Il faut que nous puissions nous-mêmes transmettre cette connaissance à des générations futures de conservateurs, de commissaires, d'archivistes, de documentalistes, etc. C'est pour nous un défi. Il y a eu de très grandes acquisitions au musée, mais This situation est, je crois, celle qui nous pousse le plus à réfléchir aux conditions d'acquisition et de conservation d'un patrimoine national. C'est pour cela que nous partageons l'information entre nous. 
Cette question de l'« autonomie » du musée me semble cruciale. Tino Sehgal envisage-t-il vraiment l'" autonomie » des musées qui acquièrent ses pièces?

L.J. Sa matière, ce sont les êtres humains et son œuvre, le choix des joueurs, les répétitions, la façon dont la pièce s'articule dans l'espace. Certaines de ses œuvres sont plus faciles à présenter, à intégrer. This situation est complexe, mais je sais que Tino Sehgal souhaite que nous devenions éventuellement autonomes.

M.F. Il est important de dire que nous ne remettons pas en question les processus d'acquisition et de présentation de ses œuvres ni son désir de pousser à leurs limites les pratiques muséologiques.

L.J. Nous allons toutes essayer, autant que possible, de vivre le processus de sélection, d'avoir une entrevue pour devenir nous-mêmes interprètes. De cette façon, nous participerons à la préparation de l'œuvre. Interpréter l'œuvre est peut-être la seule ou la meilleure façon de comprendre son fonctionnement. Mais, ce qui est problématique avec This situation, c'est qu'il faut posséder des connaissances en philosophie ou en économie.

\section{Vous avez énoncé les cinq conditions d'acquisition de} This situation, mais qu'en est-il de la pièce en tant que telle? M.F. En fait, son contenu nous est transmis oralement peu à peu. Plus nous entrons dans le processus de présentation de l'œuvre, plus nous acquérons sa connaissance.

L.J. Nous connaissons aujourd'hui quelques caractéristiques. Je peux dire que This situation est interprétée par six personnes, six intellectuels. Tino Sehgal recherche de jeunes joueurs - des doctorants ou des postdoctorants -, mais aussi des professeurs émérites, des intellectuels plus âgés issus de plusieurs champs de compétences: économie, philosophie, littérature comparée, sciences politiques, économie politique. Il ne veut pas d'historiens de l'art. Nous savons aussi qu'à chaque fois qu'un visiteur entre dans la salle, les six joueurs prononcent: «Welcome to This situation ". Puis, l'un d'entre eux dit une phrase choisie parmi une sélection de citations qui deviennent le point de départ de discussions. Ces citations sont issues de la pensée occidentale des cinq siècles derniers, de la philosophie de Marx, de Montaigne ou de Foucault, par exemple. Le but ultime est de discuter avec les visiteurs. Ce n'est pas une joute entre six intellectuels; c'est un échange qui a une base chorégraphique et orale.

\section{Vous connaissez ces citations?}

L.J. Pas encore. Cette liste sera transmise aux joueurs. Ce que je sais, c'est que tous n'ont pas à apprendre la centaine de citations. Seront-elles transmises oralement ou par courriel? Je l'ignore. 
Avez-vous déjà réfléchi à la médiation de ce travail, à sa présentation dans les communications et auprès des visiteurs?

M.F. Il n'y a aucune médiation. Tino Sehgal souhaite que les gens entrent dans le musée avec le moins d'information possible.

L.J. Il n'y a pas de cartel, pas de nom, pas de titre bien en vue. Par contre, j'aimerais que tous au musée, les gardiens, les médiateurs, les gens de la billetterie connaissent l'œuvre et ses particularités afin de pouvoir présenter la démarche de l'artiste en cas de besoin.

En terminant, que préférez-vous dans le travail de Tino Sehgal? L.J. J'éprouve beaucoup de respect pour son engagement. J'admire cette idée de pointer, de cristalliser ce qu'est l'expérience d'une œuvre d'art comme engagement de soi, de son corps, de sa tête ou de ses émotions. Ce qu'il veut faire, je pense, c'est enrichir cet engagement et le ramener à quelque chose de très simple: des gestes, des corps, de la parole. Je trouve que sa réflexion sur l'économie de l'art est brillante. On peut, au départ, se poser des questions devant ce travail, parce que ça peut paraître irrévérencieux ou provocateur, mais ce n'est pas cela. Il y a une sincérité dans sa réflexion et une compréhension de ce que sont le milieu de l'art, le musée, la performance et ses failles. Je pense, par exemple, aux images de performances qui deviennent des clichés. Tino Sehgal se demande comment éviter ces clichés. Et c'est un privilège d'avoir ce contact avec un artiste. C'est comme si nous étions dans son atelier, avec lui, et que nous le voyions choisir ses matériaux. C'est un accès incroyable.

J.B. Ce qui me fascine, c'est cette perte de l'objet. J'ai hâte de voir comment les choses vont se formaliser.

M.F. La première fois que j'ai vu les œuvres de Tino Sehgal, j'ai été fascinée. J'ai beaucoup travaillé sur la relation en art et sur la question de l'oralité. Quand je me suis plongée dans ce projet d'acquisition, j'ai compris l'importance de la transmission orale. D'un point de vue strictement institutionnel, je rejoins aussi Lesley. C'est important que le musée participe au développement du savoir et des nouvelles connaissances sur l'art, qu'il permette à l'équipe de la conservation, de réfléchir à ces nouvelles façons de faire. De savoir que l'artiste travaille avec nous, avec un lien très fort, c'est aussi stimulant. L'engagement est là.

$$
\text { 获 }
$$

Nous sommes à quelques jours de l'ouverture de l'exposition de Tino Sehgal. Nous savons que l'artiste n'a pas pu faire le voyage et que c'est plutôt l'un de ses assistants qui assure le travail de préparation. Comment se déroule ce travail?

L.J. En effet, c'est Asad Raza, qui se définit comme un "producteur " des œuvres de Tino Sehgal, qui est à Montréal depuis quelques semaines. C'est lui qui choisit les interprètes, qui organise leur horaire de jeu. Que Tino ne vienne pas, c'est dommage, mais ça arrive fréquemment. Asad et lui communiquent tous les jours. La sélection des interprètes, par exemple, a été validée par Tino. 


\section{Comment avez-vous procédé pour sélectionner ces interprètes?}

L.J. Pour Kiss, nous avons fait un appel dans les écoles et nous avons organisé des auditions. Puis, nous avons accueilli une danseuse de New York, Chloe Douglas, qui a interprété Kiss au Guggenheim. Avec Asad, ils ont choisi six couples. Pour This situation, c'est Asad qui a été le maître d'œuvre. Il a commencé par communiquer avec quelques personnes clés, des connaissances personnelles. Ça s'est fait de manière organique. Nous avons des participants des quatre universités montréalaises. Ils sont presque tous bilingues et pourront s'exprimer dans les deux langues.

\section{Pour This situation, vous avez organisé des entrevues?}

L.J. Oui. Asad a invité les gens à prendre un café pour discuter de leur champ de recherche. Asad glissait aussi quelques mots sur les enjeux et les idées philosophiques de l'œuvre, du travail de Tino. Tout cela est très fragile. Asad est extrêmement minutieux. Il faut les séduire, les intéresser, piquer leur curiosité. Il construit leur confiance comme on la construit dans une relation naissante. Il ne livre pas tout d'un coup, pas plus à eux qu'à moi d'ailleurs. Les joueurs sont rémunérés, mais c'est peu. C'est un énorme investissement de leur part.

En novembre, vous disiez ne pas connaître tous les paramètres de l'œuvre, son " contenu ", la liste des citations ou l'enchaînement de figures, par exemple. Qu'en est-il aujourd'hui?

L.J. Nous ne savons pas tout encore! Les citations, elles sont un peu le script de l'œuvre. Asad les a communiquées aux joueurs, mais personne ne les connaît toutes. Ils sont en train de les apprendre comme ils apprennent les chorégraphies, les transitions. Nous savons qu'à chaque fois qu'un visiteur entre dans la salle, tous les joueurs se tournent vers lui, prennent une grande respiration et lui souhaitent la bienvenue. L'un d'entre eux dit une citation et lance la discussion. Puis, à un moment donné, un joueur demande à un visiteur: «Qu'en pensez-vous? " À part cela, je ne sais pas. Je crois que nous allons éventuellement recevoir par courriel la totalité des citations, mais quand?

\section{Au départ, vous aviez l'intention, comme vos collègues, de} rencontrer Asad pour participer à This situation en tant qu'interprète, de manière à vivre l'expérience de l'entrevue et du jeu et ainsi favoriser la pérennité de la pièce. Qu'en est-il?

L.J. J'ai assisté au premier entretien qu'Asad a réalisé en français. L'un de mes collègues conservateurs, François LeTourneux, a eu cette rencontre. Il a une bonne connaissance de la philosophie, il est plutôt disponible et il a le désir de jouer. Nous avons donc un conservateur du musée qui va vivre l'expérience de l'intérieur. Je me suis rendu compte que ça ne pouvait pas être moi, parce je dois veiller à toute la préparation. 
Des conservateurs de l'Art Gallery of Ontario et du Fonds national d'art contemporain, en France, qui ont participé à l'acquisition d'une édition de Kiss, me disaient que des interprètes devenaient les dépositaires de l'œuvre et que c'est leur connaissance et leur travail qui assuraient la pérennité de celle-ci. Il s'agit d'une grande responsabilité. Partagez-vous leur vision? L.J. Je crois que oui. Il se pourrait qu'après les six semaines, Asad ou Chloe désignent des danseurs de Kiss ou des participants de This situation. Asad est très vigilant. Il pourrait repérer des personnes clés capables de remonter l'œuvre, ici ou ailleurs. Il conserve les noms, ce que nous allons faire aussi.

Aujourd'hui, envisagez-vous de présenter un jour This situation sans la participation de Tino Sehgal ou de l'un de ses représentants?

L.J. Je ne sais pas encore. Si nous remontons This situation, je serai peutêtre la commissaire. Et il y aura François et d'autres participants pour nous aider. Je vais leur demander d'écrire, de tenir des journaux de bord. Je peux imaginer que oui, mais ça ne sera pas la prochaine fois. Je ne crois pas que je pourrais tenir le rôle d'Asad ou de Tino et choisir les participants. Pour des œuvres plus simples comme This Is so Contemporary, oui, mais pas pour les plus complexes comme This situation, This variation ou These associations. Après la présentation, nous allons avoir le temps d'envisager cette question. Nous avons maintenant une communauté d'intellectuels qui connaissent très bien l'œuvre et c'est sans doute avec eux que nous pourrions représenter l'œuvre.

$$
\text { 获 }
$$

L'exposition de Tino Sehgal est terminée. Maintenant que vous avez expérimenté l'œuvre et suivi sa préparation, comment envisagez-vous, en tant qu'archiviste des collections, sa sauvegarde, sa conservation?

A.-M.Z. C'est une bonne question! Lors de nos premières discussions avec la galerie, nous avions compris qu'il serait possible pour le musée de devenir autonome, si une ou quelques personnes de l'institution s'engageaient activement dans le travail de préparation. Pour nous, c'était important, car nous allions débourser un bon montant pour acquérir l'œuvre, mais aussi pour la présenter et la représenter ultérieurement. Aujourd'hui, après l'avoir vue et après avoir suivi le travail de Lesley, je vois mal comment le musée pourrait devenir autonome. En fait, je me rends compte que la personne qui pourra remonter l'œuvre devra non seulement être disponible pendant plusieurs semaines, mais elle devra aussi bien comprendre la vision de l'artiste. Je pense que cette personne doit être un artiste ou, du moins, posséder une sensibilité particulière. Mais il y a un espoir. Il nous a fallu étudier et présenter plusieurs fois certaines ouvres de la collection pour arriver à bien savoir les exposer. 
Les situations construites de Tino Sehgal génèrent un conflit dans le travail de conservation, vous obligeant à sauvegarder une œuvre sans recourir à une documentation qui permet d'outrepasser l'intime et l'éphémère. Si vous produisez une documentation, vous dénaturez l'œuvre, mais si vous ne le faites pas, vous risquez de la perdre.

A.-M.Z. Oui, il y a un conflit, une friction importante. Mais quand le musée a participé à cette transaction orale, il a accepté les paramètres de l'œuvre. Étions-nous pleinement conscients de tout ce que ça impliquait? Je ne sais pas. En même temps, ce travail nous amène à collaborer, à discuter, à nous engager autrement, ce qui est bien. Je me souviens avoir assisté à une discussion de This situation qui portait sur l'économie et l'échange. À un moment, une personne a rappelé que cette œuvre faisait partie de la collection du musée et qu'elle avait été achetée. Il y a eu, en effet, une transaction économique : nous avons payé pour obtenir quelque chose. Mais pour obtenir quoi? La réponse n'est pas évidente. Le musée a notamment payé pour une œuvre qu'il souhaite présenter. Mais pour cela, il doit d'abord composer avec des contraintes importantes.

\section{Avez-vous tout de même colligé une documentation?}

A.-M.Z. Comme vous savez, nous avons noté ce qui a été dit lors la transaction orale. Nous conservons aussi le nom et les coordonnées des joueurs. Et je sais qu'au moins un joueur a tenu un journal de bord et que François et Lesley ont pris des notes que je pourrai numériser. Tout le reste, ce qui porte sur Tino Sehgal et sur ses œuvres, est disponible ailleurs, sur le Net. Nous avons eu des discussions, Josée, Lesley, Asad et moi, depuis le début de la présentation de l'œuvre, mais je me rends compte que nous ne pouvons pas écrire l'essentiel de ce qui a été échangé. Il est là notre engagement: le musée s'engage à conserver une mémoire vivante de l'œuvre et à veiller à ce qu'il y ait une transmission orale, entre conservateurs et archivistes, présents et futurs.

Il s'agit d'un véritable défi! La mémoire est une faculté qui oublie. A.-M.Z. La mémoire est quelque chose qui se transforme. La transformation peut faire partie de l'œuvre, je crois. Prenons les citations, par exemple. Vous savez que nous avons beaucoup insisté pour en obtenir la liste. Nous avons compris qu'une vingtaine avait été envoyée à quelques joueurs et que d'autres en avaient reçu des différentes. Asad a refusé de nous les communiquer. Pourquoi? Parce cela pourrait détruire l'œuvre. Au départ, j'associais ces citations à des paramètres de présentation, alors qu'aujourd'hui je comprends que ce qu'il faut conserver, c'est l'expérience de l'œuvre que nous avons eue. Il faut se rappeler son comportement. L'œuvre doit engager le public, lui demander de participer et d'échanger sur des idées. Les citations qui activeront This situation, la prochaine fois, ne seront peut-être pas toutes les mêmes. L'artiste en proposera peut-être d'autres. J'ai compris qu'elles peuvent changer avec le temps, selon les contextes. 


\section{Tout ce travail ne vous donne-t-il pas le vertige?}

A.-M.Z. Oui, mais je l'aborde comme un défi absolument magnifique. Il contredit tout ce que nous faisons de manière générale. Dans une autre perspective, qu'arriverait-il si les archives prenaient feu et que la base de données sautait complètement? Que nous resterait-il ? Notre mémoire des œuvres que nous avons côtoyées tous les jours. Je sais que je tiens un discours philosophique, mais je trouve ça beau.

Pouvons-nous imaginer que, dans cent ans, This situation aura disparu?

A.-M.Z. Si on ne la représente pas... Mais il y aura toujours quelque chose dans la base de données et dans la mémoire de quelques personnes impliquées. Je crois que nous allons continuer à la présenter. Nous avons de beaux projets. Ça augure bien. 
Amélie Giguère, translated by Allana Carlyle

\section{Acquiring This Situation by Tino Sehgal. Interviews with Josée Bélisle, Marie Fraser, Lesley Johnstone and Anne-Marie Zeppetelli from Musée d'art contemporain de Montréal}

For contemporary art, the use of fragile, unknown or ephemeral materials as well as the significant importance placed on the condition of the presentation of works, generates particular difficulties regarding the art's preservation, restoration and exhibition. Since the beginning of the 1990's, museum professionals, art historians and museologues have worked to resolve these difficulties and to understand the nature and the effects of these transformations on current museum practices. From these transformations, we see that professionals and current artists are being given new responsibilities. If, for example, curators are called to recreate an ephemeral work or to arrange elements of the installation in the space, artists are often called on to actively participate in the documentation of their work or to supervise their restorations. The fields of competence of these roles, once clearly defined, now intersect and create a space for discussion, collaboration and negotiation.

In the context of this shared responsibility, this article examines how through dialogue, curators, archivists, and artists prefer preservation, and in fact negotiate it's entry into museum collections and exhibits. Through the examination of two case studies, this article shows that the dialogue occurs at two strategic moments: Firstly during the shaping of a museum exhibition proposal and secondly in revealing the status of the various elements which make up the exhibition proposal. Based on this article review, the following questions are raised: How is the exhibition realized? How is it's renewal initiated? Which objects and documents are to be selected and defined? Are the objects collected, restored and documented works of art, archives or accessories? What is their relationship to the exhibit with which they are associated?

The first study focuses on the process of acquisition by the Musée national d'art modern in Paris at the end of the 2000s of a display by Orlan created in 1977 and entitled Action ORLAN-CORPS. MesuRage du Centre Georges Pompidou. The study demonstrates how the museum places a request to the artist, a negotiation and a sharing of information takes place, and the result is a documentary collection of the exhibition. The second study primarily examines the acquisition of a painting by Claudie Gagnon entitled Les Époux Arnolfini. This work was acquired by the Musée national des beaux-arts in Quebec also during the 2000s and was presented for the first time in 2008. The study recounts the adaptation of a tableau vivant and its possible future rendering into living form, that is the form that was originally proposed by the artist.

In both cases, these articles demonstrate that it is dialogue which allows for the identification of the constituent properties of exhibitions that define their integrity. Also, that responses to questions raised through the work of musealization become apparent during the crossing of 
preservation and creation practices. In short, the articles show that the knowledge of the status of objects, archives and documents which replace or accompany exhibitions as well as the relationships they have with exhibitions, shed light on our relationship with the work of artists and consequently play a decisive role in the preservation of their work. 